\title{
The Investigation and Management of Adenomyosis in Women Who Wish to Improve or Preserve Fertility
}

\author{
Jin-Jiao Li, ${ }^{1}$ Jacqueline P. W. Chung, ${ }^{2}$ Sha Wang, ${ }^{1}$ Tin-Chiu Li, ${ }^{2}$ and Hua Duan $\left({ }^{1}\right.$ \\ ${ }^{1}$ Department of Gynecology Minimally Invasive Center, Beijing Obstetrics and Gynecology Hospital, Capital Medical University, \\ Beijing 100006, China \\ ${ }^{2}$ Department of Obstetrics \& Gynaecology, Prince of Wales Hospital, Chinese University of Hong Kong, Shatin, Hong Kong
}

Correspondence should be addressed to Hua Duan; duanhuasci@163.com

Received 9 August 2017; Accepted 18 January 2018; Published 15 March 2018

Academic Editor: William H. Catherino

Copyright (C) 2018 Jin-Jiao Li et al. This is an open access article distributed under the Creative Commons Attribution License, which permits unrestricted use, distribution, and reproduction in any medium, provided the original work is properly cited.

\begin{abstract}
The management of adenomyosis remains a great challenge to practicing gynaecologists. Until recently, hysterectomy has been the only definitive treatment in women who have completed child bearing. A number of nonsurgical and minimally invasive, fertilitysparing surgical treatment options have recently been developed. This review focuses on three aspects of management, namely, (1) newly introduced nonsurgical treatments; (2) management strategies of reproductive failures associated with adenomyosis; and (3) surgical approaches to the management of cystic adenomyoma.
\end{abstract}

\section{Introduction}

Adenomyosis is a common benign gynaecological condition but its diagnosis and treatment remain a clinical challenge to physicians. The true incidence of adenomyosis is unknown and the prevalence varies widely due to the lack of a standardized definition and diagnostic criteria. The prevalence from previous retrospective cohort and prospective cohort observational studies is summarized in Tables 1 and 2 [1-9]. Adenomyosis also commonly occurs together with endometriosis. Di Donato et al. [10] showed a prevalence of $21.8 \%$ in women undergoing surgery for endometriosis. They also showed an association with parous women, increasing age, dysmenorrhea intensity, and presence of deep infiltrating endometriosis.

Adenomyosis is best defined by Bird in 1972 as "the benign invasion of endometrium into the myometrium, producing a diffusely enlarged uterus which microscopically exhibits ectopic non-neoplastic, endometrial glands and stroma surrounded by the hypertrophic and hyperplastic myometrium" [11].

\section{Pathogenesis}

The exact pathogenesis of adenomyosis remains debatable. The diagnosis of adenomyosis is made when ectopic endometrial implants are found within the myometrium of the uterus. The most common and widely accepted theory involves the downward invagination of the endometrial basalis layer into the myometrium due to either myometrial weakness or altered immunologic activity leading to disruption of the endometrial-myometrial interface, also known as the "junctional zone (JZ)" [12]. Leyendecker et al. [13] showed that uterine auto-traumatisation and the initiation of the mechanism of tissue injury and repair (TIAR) as the primary cause for adenomyosis development based on their method of "visualization" by transvaginal ultrasound (TVS) and cinematographic magnetic resonance imaging (MRI). Their group showed the archimetral compression from the neometral contraction at the onset of menstruation causes high intrauterine pressure, leading to rupture of the archimyometrium at cornual angles. Thus, fragments of the basal endometrium are then detached and deposited into the myometrial wall where they develop into endometriotic cysts. In addition, as the basal stromal cells at the fundo-cornual raphe are chronically over stretched, it initiates the TIAR mechanism and development of an adenomyoma. Other theories include de novo development from embryonic-misplaced pluripotent Mullerian remnants or invagination along the intramyometrial lymphatic system or displaced bone marrow stem cells [14]. 
TABLE 1: Prevalence of adenomyosis after hysterectomy specimens for various gynaecological conditions (from retrospective cohort studies).

\begin{tabular}{|c|c|c|c|c|c|}
\hline Study & $\begin{array}{c}\text { Vercellini et al. } \\
1995[1]\end{array}$ & $\begin{array}{c}\text { Vavilis et al. } \\
1997[2]\end{array}$ & $\begin{array}{c}\text { Seidman and } \\
\text { Kjerulff1996 [3] }\end{array}$ & $\begin{array}{c}\text { Parazzini et al. } \\
1997[4]\end{array}$ & $\begin{array}{c}\text { Bergholt et al. } \\
2001[5]\end{array}$ \\
\hline Number of cases $(n)$ & 1334 & 594 & 1252 & 707 & 549 \\
\hline Adenomyosis (\%) & 25 & 20 & $12-58$ & 21 & $10-18$ \\
\hline Uterine fibroid & 23 & 21 & & 15 & \\
\hline Genital prolapse & 26 & 26 & & 30 & \\
\hline Ovarian cyst & 21 & 18 & & 30 & \\
\hline Cervical cancer & 19 & 18 & & 25 & \\
\hline Endometrial cancer & 28 & 16 & & & \\
\hline Ovarian cancer & 28 & 21 & & & \\
\hline
\end{tabular}

\section{Diagnosis}

Histological examination is the gold standard in the diagnosis of adenomyosis, even though the exact histological criteria have not been universally agreed. One accepted criterion is the presence of endometrial tissue more than $2.5 \mathrm{~mm}$ below the endomyometrial junction or a JZ thickness of more than $12 \mathrm{~mm}$ [15]. The modification of the uterine structure may range from thickening of the $\mathrm{JZ}$ of $>12 \mathrm{~mm}$ to nodular or diffuse lesions involving the entire uterus. Thus, adenomyosis is classified to "diffuse adenomyosis" where endometrial deposits are found dispersed within the myometrium or "focal adenomyoma" where the endometrial deposits are more localized at one site within the uterine wall as a confined lesion [14].

Apart from the findings of these ectopic endometrial tissues within the myometrium, smooth muscle changes like hyperplasia are often found. Ultrastructural differences between smooth muscle cells from adenomyosis and normal uterus were found with myocytes showing cellular hypertrophy, differences in cytoplasmic organelles, nuclear structures, and intercellular junctions [15]. The myocytes in adenomyosis also lack the cyclical changes present in myocytes of the normal uterus [16].

\section{Cystic Adenomyoma}

Rarely, adenomyosis may present as a cystic lesion lined with endometrial tissue and surrounded by myometrial tissue when it is called "cystic adenomyoma." Juvenile cystic adenomyoma (JCA) is a subgroup of cystic adenomyoma that commonly occurs in adolescents or women $<30$ years of age and is not associated with diffuse adenomyosis. Takeuchi et al. [17] proposed the following diagnostic features of juvenile cystic adenomyoma (JCA): (1) age < 30 years; (2) cystic lesion of $>1 \mathrm{~cm}$ in diameter independent of the uterine lumen and covered by hypertrophic myometrium on diagnostic images; and (3) association with severe dysmenorrhea. They found that laparoscopic excision of the lesion demonstrated significant improvement of dysmenorrhea in these cases.

\section{Presentations}

The classic presentation of adenomyosis is heavy, painful menstrual bleeding, typically occurring in multiparous women between 40 and 50 years of age [14]. Heavy menstrual bleeding is present in up to $40-60 \%$ of patients, which may be due to the enlarged endometrial surface area or the increased vascularity of the endometrium [14]. Dysmenorrhea occurs in $15-30 \%$ of patients, which may be related to the swelling of endometrial tissue within the myometrium or increased production of prostaglandin within the myometrium [18]. Both the amount of bleeding and degree of pain were shown to be significantly correlated with the degree of myometrial invasion [18]. Other presenting features include chronic pelvic pain, dyspareunia, and the finding of an enlarged uterus in an asymptomatic subject. Women with adenomyosis had been shown to have a decreased quality of life [19], up to $33 \%$ of patients may be asymptomatic, and the diagnosis of up to $30 \%$ of patients was only made by histology following a hysterectomy [20].

There is also increasing evidence to show an association between infertility and adenomyosis [21]. Several mechanisms may be involved, including impairment of sperm transport [7], aberrant uterine contractility [22], alterations of adhesion molecules, cell proliferation, apoptosis, and free radical metabolism $[15,23]$. Adenomyosis is also speculated to be a cause of recurrent implantation failure during IVF treatment [24].

\section{Investigation}

6.1. Two-Dimensional Ultrasound (USG). Two-dimensional (2D) transabdominal USG may reveal uterine enlargement or asymmetric thickening of the anterior and posterior myometrial walls. However, transabdominal USG is often not accurate enough in diagnosing adenomyosis as it fails to provide sufficient image resolution for visualization of the myometrium. Therefore, $2 \mathrm{D}$ transvaginal USG is often the first-line investigation. In a review performed by Reinhold et al., it was shown that transvaginal USG had a sensitivity of $80-86 \%$, specificity of $50-96 \%$, and an overall accuracy of $68-86 \%$ in diagnosing diffuse adenomyosis [25].

USG features of adenomyosis include the presence of three or more sonographic criteria: heterogeneity, increased echogenicity, decreased echogenicity, and anechoic lacunae or myometrial cysts [26]. In contrast to uterine fibroids, adenomyoma has a more elliptical shaped lesion with poorly defined borders, no calcifications, or edge shadowing. In 


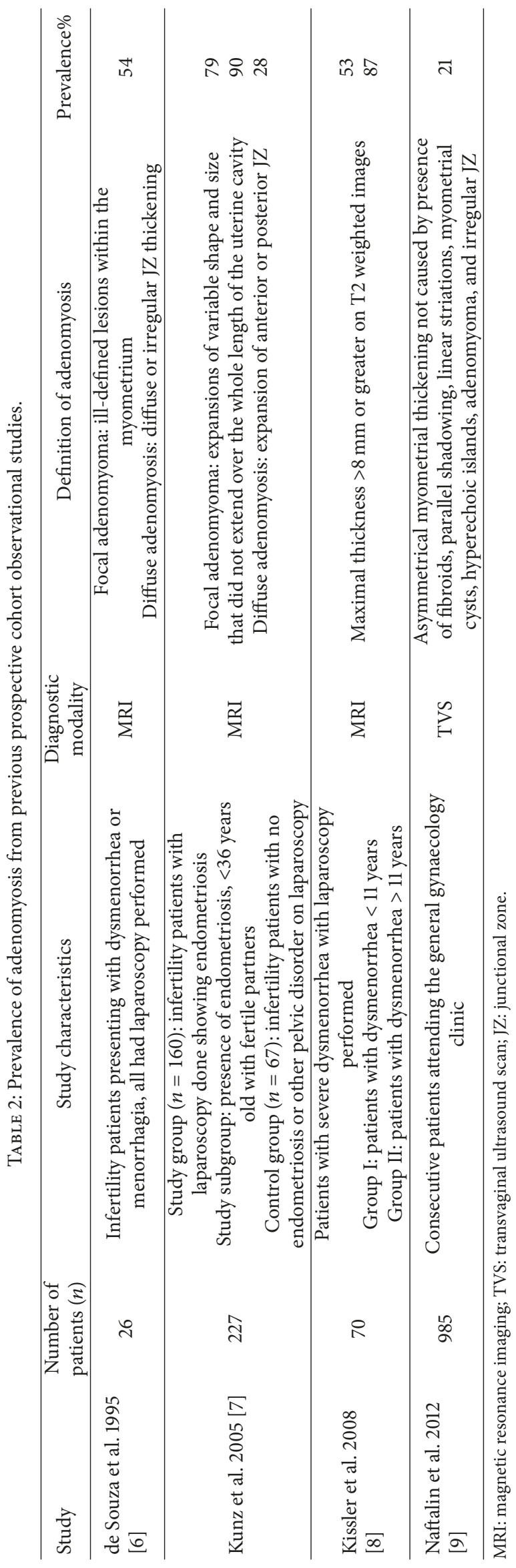


doubtful cases, Doppler sonography may be helpful in that blood vessels in the case of adenomyoma usually follow their normal vertical course in the myometrial areas while in the case of uterine fibroid, blood vessels are usually located in the periphery [27].

Sonographic diagnosis of adenomyosis is not always easy but the consensus statement and recommendation published by the MUSA (Morphological Uterus Sonographic Assessment) group on how sonographic features of adenomyosis should be described and measured should help to improve the diagnostic accuracy [28].

6.2. Three-Dimensional Ultrasound. Three-dimensional (3D) USG improves diagnostic accuracy of adenomyosis as it allows better imaging of the JZ [29]. The JZ is often visible as a hypoechogenic subendometrial halo which is composed of longitudinal and circular closely packed smooth muscle fibers. Upon 3D USG, adenomyosis is characterized by a thickened or irregular JZ [30]. Ahmadi and Haghighi showed the accuracy of 3D transvaginal USG in the diagnosis of adenomyosis to be $80 \%$ and a positive predictive value of $95 \%$ based on the detection of an irregular JZ on coronal plane [31]. Exacoustos et al. [30] analyzed a total of 72 premenopausal patients with $2 \mathrm{D}$ and $3 \mathrm{D}$ transvaginal USG before hysterectomy. In the study, the histological prevalence of adenomyosis was $44.4 \%$. Their group agrees that the coronal section of the uterus obtained by $3 \mathrm{D}$ transvaginal USG allows accurate evaluation and measurement of the JZ and its alteration shows good diagnostic accuracy for adenomyosis. They showed that the presence of myometrial cysts was the most specific 2D transvaginal USG feature with specificity of $98 \%$ and accuracy of $78 \%$ while heterogeneous myometrium was the most sensitive feature with a sensitivity of $88 \%$ and accuracy of $75 \%$. As for 3D transvaginal USG, with a JZ difference of more than or equal to $4 \mathrm{~mm}$, JZ infiltration and distortion had a high sensitivity of $88 \%$ and the best accuracy of $85 \%$ and $82 \%$, respectively. The overall accuracy of diagnosing adenomyosis for $2 \mathrm{D}$ and $3 \mathrm{D}$ transvaginal USG was $83 \%$ and $89 \%$, sensitivity was $75 \%$ and $91 \%$, specificity was $90 \%$ and $88 \%$, positive predictive value was $86 \%$ and $85 \%$, and negative predictive value was $82 \%$ and $92 \%$, respectively. 3D USG also has the advantage of allowing storage of the images with subsequent offline manipulation and interpretation.

6.3. Magnetic Resonance Imaging. Magnetic resonance imaging (MRI) is the gold standard imaging modality for assessing the JZ in the evaluation of adenomyosis [32]. The common features of adenomyosis on MRI include (1) thickening of the JZ, JZ thickness $\geq 12 \mathrm{~mm}$, or irregular junctional thickness with a difference of $>5 \mathrm{~mm}$ between the maximum thickness and the minimum thickness, (2) an ill-defined area of low signal intensity in the myometrium on T2-weighted MR images, and (3) islands of ectopic endometrial tissue identified as punctate foci of high signal intensity on T1-weighted image [32-34]. However, MRI is expensive and may not be readily available in every unit. Moreover, Reinhold et al. [33] prospectively studied 119 patients undergoing hysterectomy and compared findings between TVS and MRI. The study
TABLE 3: Accuracy of TVS and MRI for the noninvasive diagnosis of adenomyosis.

\begin{tabular}{lcc}
\hline & TVS & MRI \\
\hline Sensitivity & $72 \%$ & $77 \%$ \\
Specificity & $81 \%$ & $89 \%$ \\
Positive likelihood ratio & 3.7 & 6.5 \\
Negative likelihood ratio & 0.3 & 0.2 \\
\hline
\end{tabular}

TVS: transvaginal ultrasound scan; MRI: magnetic resonance imaging.

showed that there was no significant difference in sensitivity and specificity between the two groups. Champaneria et al. [34] also performed a systematic review comparing test accuracy between USG and MRI for the diagnosis of adenomyosis. Their study findings are summarized in Table 3. They agreed that both TVS and MRI show high levels of accuracy for the noninvasive diagnosis of adenomyosis. However, we believe MRI may be particularly useful in the assessment of focal adenomyoma and provides important information on whether surgery should proceed.

6.4. Shear Wave Elastography. A recent study also showed that using Aixplorer (Supersonic Imagine, France) scanner with application of shear wave elastography during transvaginal scanning may improve diagnostic accuracy of adenomyosis [35]. This study found that adenomyosis was associated with a significant increase of the myometrial stiffness estimated with shear wave elastography. Further studies are required to verify the clinical usefulness of such an approach.

6.5. Hysterosalpingography. Hysterosalpingography is seldom used to diagnose adenomyosis. However, in patients undergoing infertility assessment, the occasional finding of spiculations measuring $1-4 \mathrm{~mm}$ in length, arising from the endometrium towards the myometrium, or a uterus with the "tuba erecta" finding may be suggestive of adenomyosis [36].

6.6. Hysteroscopy. Several hysteroscopic appearances have been found to be associated with adenomyosis, including irregular endometrium with endometrial defects or superficial openings, hypervascularization, strawberry pattern, or cystic haemorrhagic lesions [37]. Nevertheless, there is limited data available on the diagnostic accuracy of these various features.

6.7. Hysteroscopic and Laparoscopic Myometrial Biopsy. In 1992, McCausland [38] showed that myometrial biopsy is helpful to diagnose adenomyosis. The study found that the depth of adenomyosis was correlated with the severity of menorrhagia. Of the 90 patients studied, 50 patients had normal hysteroscopy in which $55 \%$ of them had significant adenomyosis (greater than $1 \mathrm{~mm}$ ) when compared to controls $(0.8 \mathrm{~mm})$. In that study, it was suggested that minimal adenomyosis may be treated definitively by endometrial ablation while deep adenomyosis should be treated by hysterectomy. They also showed that endometrial glands left under a scar could not only bleed and cause pain but also have malignant 
potential. The authors suggested routine myometrial biopsy at the time of operative hysterectomy should be considered. However, Darwish et al. [39] showed hysteroscopic myometrial biopsies using rigid biopsy forceps to be inadequate and did not recommend its use. Popp et al. [40] showed that the sensitivity of a single myometrial biopsy in diagnosing adenomyosis ranged from 8 to $18.7 \%$, while the specificity was $100 \%$ among 680 biopsy specimens in 68 surgically removed uterus using automatic cutting needle sampling. Gordts et al. [41] recommended the use of hysteroscopic guided biopsy for the diagnosis of adenomyosis using a new device, the UteroSpirotome. It can also be used under ultrasound guidance to get access to small cystic adenomyoma lesions.

6.8. Laparoscopic Myometrial Biopsy. In a prospective, nonrandomized study conducted by Jeng et al. [42] evaluating 100 patients with clinical signs and symptoms strongly suggestive of adenomyosis, the sensitivity of myometrial biopsy was $98 \%$ and the specificity $100 \%$; the positive predictive value was $100 \%$ and the negative predictive value $80 \%$, which were superior to those of transvaginal sonography, serum CA125 determination, or the combination of both. The group suggested that laparoscopy-guided myometrial biopsy is a valuable tool in the diagnosis of diffuse adenomyosis in women presenting with infertility, dysmenorrhea, or chronic pelvic pain.

\section{Management}

As in the case of endometriosis, the management strategy of adenomyosis depends primarily on the presenting symptom and whether it is associated with reproductive failure.

\subsection{Management of Menstrual Symptoms}

7.1.1. Medical Treatment. Medical treatment for adenomyosis is similar to those given for endometriosis. Apart from symptomatic relief, hormonal treatment mainly works by inhibition of ovulation, cessation of menses, improving the hormonal milieu, and causing decidualization of the endometrial deposits.

Analgesic. Nonsteroidal anti-inflammatory drugs (NSAIDs) work by inhibiting the cyclooxygenase (COX-1 and COX-2) and decreasing the production of prostaglandins. NSAIDs have been proved to be effective in treatment of primary dysmenorrhea by Gambone et al. [43]. It is usually the firstline treatment for symptomatic pain relief for adenomyosis.

Oral Contraceptive Pills (OCPs). Combined oral contraceptive pills work by inhibiting ovulation by suppressing the release of gonadotrophins. Many studies have shown that they are effective in the treatment of dysmenorrhea. A prospective observational trial showed that continuous low-dose OCP were more effective than cyclical low-dose OCP in controlling symptoms in patients after surgical treatment for endometriosis [44]. Mansouri et al. [45] have shown regression of adenomyosis on MRI after using oral contraceptive pills for 3 years in adolescents with adenomyosis presenting with chronic pelvic pain.
Danazol. Danazol is an isoxazol derivative of 12 alpha-ethinyl testosterone. It causes a hypogonadic state and thus is widely used for treatment of endometriosis and abnormal uterine bleeding [46]. However, data on its use in adenomyosis remains limited. This may be due to its unwanted adverse effects after systemic treatment. In 2000, Igarashi et al. [47] reported a novel conservative medical therapy for uterine adenomyosis with a danazol-loaded intrauterine device in 14 women. During insertion of the danazol-loaded IUD, there was complete remission of dysmenorrhea in 9 patients, reduction in 4 , and no change in 1 patient. There was complete remission of hypermenorrhea in 12 patients and no change in 2. Nine out of 14 patients also showed reduction in the maximum thickness of the myometrium as measured by MRI. However, further studies are required to confirm the clinical usefulness of the treatment.

Dienogest. Dienogest is a selective synthetic oral progestin that combines the pharmacological properties of 17-alphaprogesterone and 19 nor-progesterone with pronounced local effect on endometrial tissue. Dienogest has been shown to be effective in the treatment of endometriosis associated pelvic pain. A prospective clinical trial has shown dienogest to be a valuable alternative to depot triptorelin acetate for treatment of premenopausal pelvic pains in women with uterine adenomyosis. The study included a total of 41 patients with adenomyosis with pelvic pain and menorrhagia. The patients were allocated to receive oral dienogest $(2 \mathrm{mg} /$ day $)$ or triptorelin acetate $(3.75 \mathrm{mg} / 4$ weeks) for 16 weeks. Both treatments were highly effective in treatment of dysmenorrhea, dyspareunia, and chronic pelvic pain associated with adenomyosis, although triptorelin acetate appeared superior to dienogest in controlling menorrhagia [48].

Levonorgestrel-Releasing Intrauterine Device (LNG-IUD). LNG-IUD is an intrauterine device, which release 20 micrograms of levonorgestrel per day. It has been shown to be an effective treatment for abnormal uterine bleeding. LNG-IUD acts locally and causes decidualization of the endometrium and adenomyotic deposits. LNG-IUD alleviates dysmenorrhea by improving uterine contractility and reducing local prostaglandin production within the endometrium. LNG-IUD appears to be an effective method in relieving dysmenorrhea associated with adenomyosis [49] and more effective than the combined OC pill [50], improved the quality of life [19], and appears to be a promising alternative treatment to hysterectomy.

LNG-IUD may be used in conjunction with other treatment modalities such as GnRH analogue [51] or transcervical resection of the endometrium (TCRE) [52]. In the latter study, it was found that TCRE combined with LNG-IUD was more effective in reducing menstrual flow compared with the LNGIUD alone although there was no significant difference in the amount of pain reduction between the two treatment strategies.

GnRH Agonists. GnRH agonists are effective in alleviating dysmenorrhea and relieving menorrhagia associated with adenomyosis [53]. However, due to the undesirable 
climacteric side effects and risk of osteoporosis, treatment with GnRH agonists is usually restricted to a short duration of 3-6 months although the duration of use may be extended if add-back estrogen therapy is employed [54]. Discontinuation of treatment usually leads to regrowth of the lesions and recurrence of symptoms.

Selective Estrogen Receptor Modulator (SERM). Selective estrogen receptor modulators like tamoxifen or raloxifene have been tried in the treatment of endometriosis [54] based on observations that SERMs may reduce endometriosis lesion in mouse [55]; however, their value in the treatment of adenomyoma has not been formally explored.

Aromatase Inhibitors. Like endometriosis, adenomyotic deposits are estrogen-dependent. Aromatase inhibitors inhibit the conversion of estrogen from androgens, thereby lowering the synthesis of estrogen. A prospective randomized controlled study found that the efficacy of aromatase inhibitors (letrozole $2.5 \mathrm{mg}$ /day) in reducing the volume of adenomyoma as well as improving adenomyosis symptoms was similar to that of GnRH agonists (goserelin $3.6 \mathrm{mg} / \mathrm{month}$ ) [56]. Kimura et al. also reported on the combined use of aromatase inhibitors with $\mathrm{GnRH}$ agonist with good results in a 34-year-old woman with severe uterine adenomyosis who wished to preserve fertility [57]. They found a reduction in uterine volume of $60 \%$ after 8 weeks of treatment as determined by magnetic resonance imaging and ultrasound.

Ulipristal Acetate. Ulipristal acetate (UPA) is a potent selective progesterone receptor modulator. There is good evidence to suggest that it can be used to shrink fibroid and control menorrhagia $[58,59]$. It is possible that it may be similarly effective in the treatment of adenomyoma but literature data is lacking.

Antiplatelet Therapy. There is new evidence to suggest a role of antiplatelet therapy in treating adenomyosis. Emerging evidence suggests that endometriotic lesions are wounds undergoing repeated tissue injury and repair (ReTIAR), and platelets induce epithelial-mesenchymal transition (EMT) and fibroblast-to-myofibroblast transdifferentiation (FMT), leading ultimately to fibrosis. Adenomyotic lesions are thought to have similar pathogenesis to that of endometriosis. A recent study in mice suggests that antiplatelet treatment may suppress myometrial infiltration, improve generalized hyperalgesia, and reduce uterine hyperactivity [60].

7.1.2. Uterine Artery Embolization. Uterine artery embolization (UAE) has been used to treat symptomatic fibroids since the 1990s. There is increasing evidence to suggest that it is also effective in the treatment of management of adenomyosis. In a review of 15 studies including 511 women with adenomyosis, Popovic et al. found [61] significant clinical and symptomatic improvement in seventy-five percent of subjects at short- and long-term follow-up. A recent retrospective observational study of 252 patients who underwent UAE with up to five years of follow-up showed that improvement in dysmenorrhea and menorrhagia are more likely to occur in vascular lesions [62].

7.1.3. High Intensity Focused Ultrasound. High intensity focused ultrasound (HIFU) is another nonsurgical treatment for uterine fibroids that focuses high intensity ultrasound in the target lesion causing coagulative necrosis and shrinkage of the lesion. Both MRI and USG can be used for guidance for the procedure. MRI has better real time thermal mapping during the HIFU treatment. Yet, ultrasound guided HIFU is less costly and offers real time anatomic monitoring imaging and a grey scale change during treatment represents a reliable indicator in treatment response. It is effective in both focal and diffuse lesions $[63,64]$. Ultrasound guided HIFU was shown to be technically successful in up to $94.6 \%$ of patients in a review of 2549 patients among 10 different centers with symptomatic adenomyosis [65].

7.1.4. Endomyometrial Ablation or Resection. There is limited report on the use of laparoscopic or hysteroscopic endometrial in treating adenomyosis in the literature. The success rate of myometrial electrocoagulation ranges from 55 to $70 \%$ as reported [66]. Wood [67] reported success in 4 out of 7 patients who underwent myometrial electrocoagulation, while Phillips et al. [68] had 7 out of 10 patients with symptomatic adenomyosis diagnosed by MRI treated with laparoscopic bipolar coagulation, having significant reduction or resolution of dysmenorrhea or heavy menstrual bleeding.

7.1.5. Hysterectomy. Hysterectomy is the definitive treatment option for intractable symptomatic adenomyosis when medical or other conservative treatments have failed to control the symptoms. Patients undergoing hysterectomy for adenomyosis should be advised of an increased risk of bladder injury and persistent pelvic pain. Furuhashi et al. [69] reviewed 1246 vaginal hysterectomies and found that patients undergoing vaginal hysterectomy for adenomyosis have increased risk of bladder injury compared with those performed for leiomyoma (2.3\% versus $0.7 \%)$. It may be a result of difficulty in identifying the supravaginal septum and the vesicovaginal or vesicocervical planes. Several studies have reported on persistent pelvic pain after hysterectomy for adenomyosis [70]. Once a decision to proceed with hysterectomy has been made, the possibility of oophorectomy should be discussed. In general, it is not considered necessary to routinely remove the ovaries in premenopausal women $[71,72]$, but it may be indicated in women who suffer from cyclical symptoms, with concomitant ovarian endometriosis, or who are considered to have an increased risk of developing ovarian cancer, including those with a family history of the condition. Interestingly, a recent population-based study by Kok et al. [73] suggested that the risk of developing ovarian cancer in women with newly diagnosed adenomyosis is increased by 4-5-fold. If the finding is confirmed, there is a strong case to consider prophylactic oophorectomy at the time of hysterectomy for adenomyosis in premenopausal women. 
7.2. Reproductive Failure. Several studies have shown that adenomyosis is associated with a negative impact on the success rate of IVF. In a recent meta-analysis conducted by Vercellini et al. [74], adenomyosis was associated with a $28 \%$ reduction in the likelihood of a clinical pregnancy in infertile women who underwent IVF/ICSI with autologous oocytes. Patients with adenomyosis were found to have higher chances of miscarriage, independent of oocyte or embryo quality. Thalluri and Tremellen [75] also showed that the adenomyosis was associated with a significant reduction in successful implantation of good-quality embryos in patients undergoing IVF treatment (viable clinical pregnancy rate $23.6 \%$ versus $44.6 \%$ among those who did not have adenomyosis, $P=0.017$ ).

Puente et al. [76] performed a cross-sectional study of 1015 patients prior to assisted conception treatment. They found that the prevalence of adenomyosis was $24.4 \%$ in women aged $\geq 40$ years and $22 \%$ in women aged $\leq 40$ years. The prevalence of adenomyosis was found to be higher in those with recurrent pregnancy loss (38.2\%) and previous ART failure (34.7\%) when compared with those who did not (22.3\% and $24.4 \%$, respectively). They also found that 4 out of 5 patients had the diagnosis missed in earlier transvaginal ultrasonography.

The use of short-term GnRH agonists to shrink the size of the adenomyosis lesion has been shown to improve conception rate within 6 months of cessation of $\mathrm{GnRH}$ agonist therapy $[77,78]$.

In women with adenomyosis planning to undergo IVF treatment, the following management strategies should be considered.

7.2.1. GnRH Analogue Therapy before In Vitro Fertilization. Several studies have shown that pretreatment with $\mathrm{GnRH}$ analogue before IVF treatment improved pregnancy outcome. Zhou et al. [79] analyzed the clinical efficacy of leuprorelin acetate in treatment of uterine adenomyosis with infertility. They found that, after 2-6 months of leuprorelin acetate therapy, the mean uterine volume was significantly reduced from $180 \pm 73 \mathrm{~cm}^{3}$ to $86 \pm 67 \mathrm{~cm}^{3}$, leading to an improvement in embryo implantation and clinical pregnancy rates.

7.2.2. Stimulation Protocol. In women without pre-IVF $\mathrm{GnRH}$ analogue therapy as described above, long $\mathrm{GnRH}$ analogue protocol should be considered as it helps to induce decidualization of the adenomyotic deposits rendering the disease inactive. Tao et al. [80] showed that GnRH antagonist protocol appears to be inferior to $\mathrm{GnRH}$ agonist long protocol cycle, and the latter appeared to be associated with increased pregnancy and decreased miscarriage rates.

7.2.3. Two-Staged In Vitro Fertilization. In women with adenomyosis, a two-staged in vitro fertilization could be considered. Patients can undergo ovarian stimulation, oocyte retrieval, and fertilization followed by frozen-thawed embryo transfer (FET) at a later stage. Prior to the FET, GnRH analogue suppression therapy for 3 months or so leads to shrinkage of the adenomyosis. FET in the first HRT cycle following GnRH analogue suppression therapy, before the adenomyosis lesion regrows to its pretreatment size and exerts its adverse impact on implantation, may improve the result.

7.2.4. Mock Embryo Transfer. Performing a mock embryo transfer is desirable in women with adenomyosis, as it may help to assess the uterine cavity length and position, choose the correct transfer catheter, and alert the clinicians any extra precautions (e.g., use of tenaculum or cervical dilatation). Mock embryo transfer is particularly desirable in those with an enlarged uterus or distorted uterine cavity.

7.2.5. Single Embryo Transfer. Adenomyosis has been reported to be associated with increased incidence of preterm delivery, preeclampsia, and second trimester miscarriage when compared with the control group [81]. Consequently, multiple pregnancies should be avoided and so single embryo transfer should be advised. Women who had adenomyomectomy prior to IVF should also be advised to have SET to avoid multiple pregnancy with a view to minimize the risk of scar rupture.

7.2.6. HRT Protocol in Frozen-Thawed Embryo Transfer (FET) Cycle. GnRH agonist pretreatment to suppress the pituitary ovarian axis prior to hormone replacement therapy to prepare the endometrium in FET cycles appeared to improve the outcome compared with hormone replacement therapy without downregulation. In a study including 339 patients with adenomyosis, 194 received long-term GnRH agonist plus HRT (downregulation + HRT) and 145 with HRT alone. The clinical pregnancy, implantation, and ongoing pregnancy rates in the downregulation and HRT group were significantly higher than that of the HRT alone group, being $51.35 \%$ versus $24.83 \%, 32.56 \%$ versus $16.07 \%$, and $48.91 \%$ versus $21.38 \%$, respectively [82].

7.2.7. Uterine Contractility and Atosiban Therapy. Several functional studies showed that excessive uterine contractility ( $>5$ contractions per minute) has been demonstrated in approximately $30 \%$ of patients undergoing embryo transfer and this may have a significant adverse impact on subsequent embryo implantation and clinical pregnancy rates [83]. The incidence of abnormal contractility appeared to be higher in women with adenomyosis [84] which may in part explain the higher incidence of reproductive failure observed in this group of women. Although recent evidence suggests that the routine use of atosiban therapy does not improve the outcome [85], it is possible that the use of atosiban in a selected group of women with aberrant uterine contractions during embryo transfer may improve the outcome. Ideally, women with adenomyosis should be screened for abnormal uterine contractions during ET; if the results are abnormal atosiban therapy should be discussed; alternatively, the possibility of empirical atosiban therapy in women with adenomyosis and recurrent implantation failure could be considered.

7.2.8. Recurrent Implantation Failure. Recurrent implantation failure is diagnosed when there is failure to achieve a 


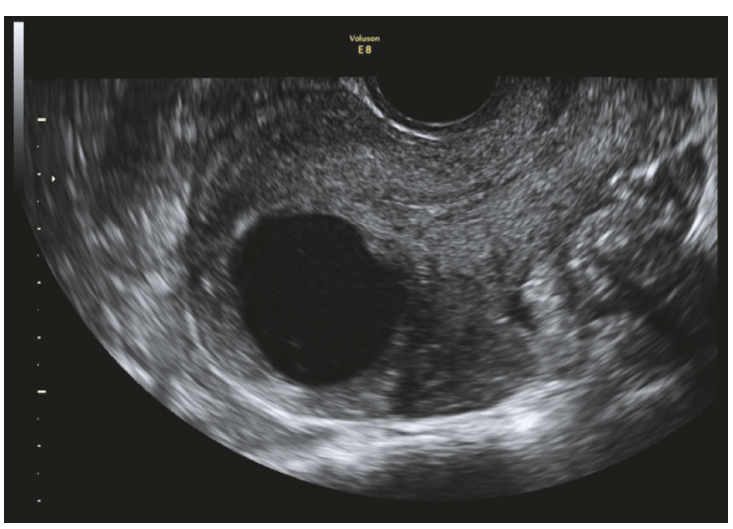

(a)

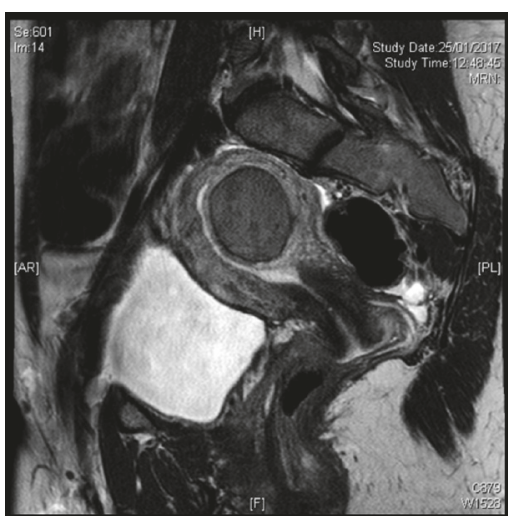

(b)

FIGURE 1: (a) Ultrasound and (b) MRI appearance of a cystic adenomyoma.

clinical pregnancy after transfer of at least four good-quality embryos in a minimum of three fresh or frozen cycles in a woman under the age of 40 years [86]. It is known that adenomyosis is associated with recurrent implantation failure [24]. Women with recurrent implantation failure should be offered 3D scan or MRI to establish if there is adenomyosis; if adenomyosis is present, the above management strategies should be adopted to improve the outcome.

7.2.9. Uterine Sparing Conservative Surgery. Surgery is seldom required for women prior to IVF treatment, the indication being (1) well-defined adenomyoma more than $5 \mathrm{~cm}$ and (2) recurrent miscarriage or recurrent implantation failure after IVF. A retrospective cohort study performed by Kishi et al. [87] involving 102 women showed that laparoscopic adenomyomectomy was beneficial for women who experienced IVF treatment failures if they were $<39$ years old but not for patients aged 40 years or more. No benefit of uterine sparing surgery is seen for those older patients aged 40 or above. Grimbizis et al. [88] reviewed the current literature and described three main categories of uterine sparing surgical treatment, including complete excision by adenomyomectomy; cystectomy or partial excision cytoreductive surgery; and nonexcisional techniques including uterine artery ligation, electrocoagulation of myometrium, resection, and ablation. The review concluded that uterine sparing treatment of adenomyosis appears feasible and effective. After complete excision, the dysmenorrhea reduction, menorrhagia control, and pregnancy rate were $82.0 \%, 68.8 \%$, and $60.5 \%$, respectively. After partial excision, the dysmenorrhea reduction rate was similar at $81.8 \%$, although menorrhagia control and pregnancy rate were slightly reduced to $50.0 \%$ and $46.9 \%$, respectively.

7.3. Hysteroscopic Surgery. Just as it is now possible to remove intramural myoma with refined hysteroscopic techniques, hysteroscopic adenomyomectomy may also be possible in selected cases, especially when the adenomyoma is $<5 \mathrm{~cm}$ or when it protrudes into the uterine cavity. However, hysteroscopic adenomyomectomy should always be carried out under USG guidance. A minimal safety margin of
$5 \mathrm{~mm}$ between the serosa and adenomyoma is considered necessary to avoid the risk of uterine perforation although the safety margin may sometimes increase after part of the lesion has been removed and the uterine contractions which follow help to push the adenomyoma further towards the cavity. Pretreatment with 3-month course of GnRH agonist beforehand can help reduce the vascularity and bleeding during the operation. Sometimes, it may also help to push the adenomyoma towards the uterine cavity due to the reduction of uterine volume.

The location of the adenomyoma should be clearly defined before the start of the procedure. Using a lower perfusing pressure, say at $40 \mathrm{mmHg}$ instead of the usual 90-100 $\mathrm{mmHg}$, may allow a slight bulge of the adenomyoma into the cavity to be visualized. Vasopressin, a potent vasoconstrictor, may be injected into the uterus by using an oocyte retrieval needle [89] to result in contraction of the uterus and reduce bleeding. Afterwards the endometrium and the myometrium overlying the adenomyoma can be incised using a cutting loop or needle or dissected with the use of a pair of scissors, following which the adenomyoma is removed by the cutting loop or a pair of grasping forceps coupled with twisting actions, separating it from the underlying myometrium. The latter step may be achieved with the use of Hysteroscopy Endo-Operative System (HEOS) [90], which allows both mechanical and electrosurgical instruments to be used. Complete removal of the adenomyoma may be difficult. A repeat surgical procedure may be required from time to time.

Cystic adenomyoma is a special category of adenomyoma. Figures 1(a) and 1(b) show the ultrasound and MRI appearance of a cystic adenomyoma. At the beginning of the hysteroscopic operation, the adenomyoma did not appear to bulge into the cavity (Figure 2(a)), but upon lowering the perfusing pressure, the cystic adenomyoma was seen bulging into the cavity (Figure 2(b)), which permits the precise location of the lesion to be identified. In this particular case, a longitudinal incision was made over the adenomyoma, draining a large amount of blood clots from the cystic adenomyoma. In this case, initial attempts to dissect the cystic adenomyoma away from the myometrium (Figure 2(c)) had 


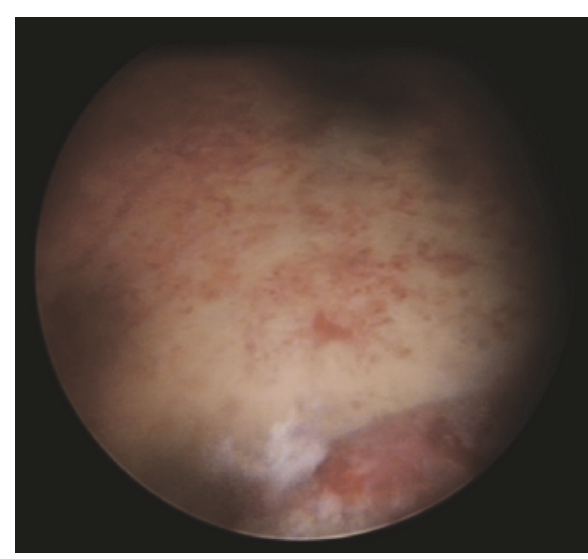

(a)

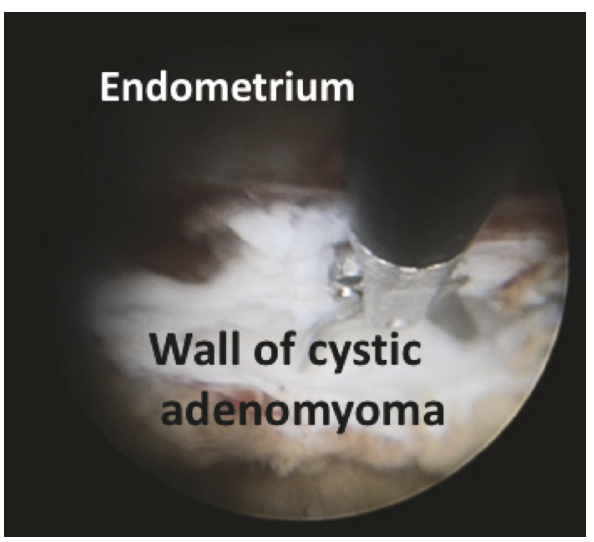

(c)

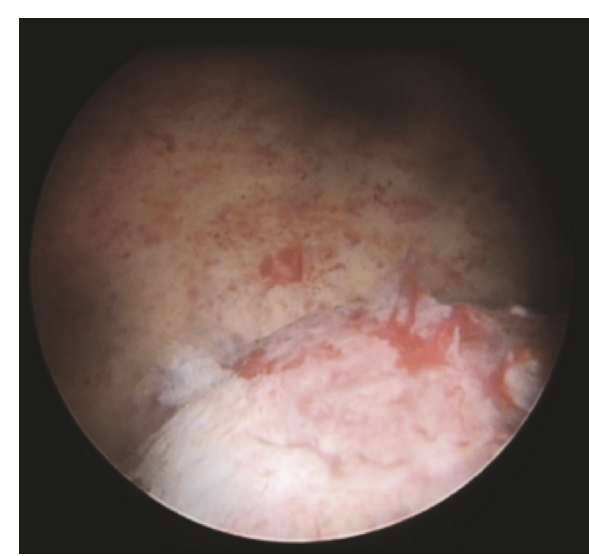

(b)

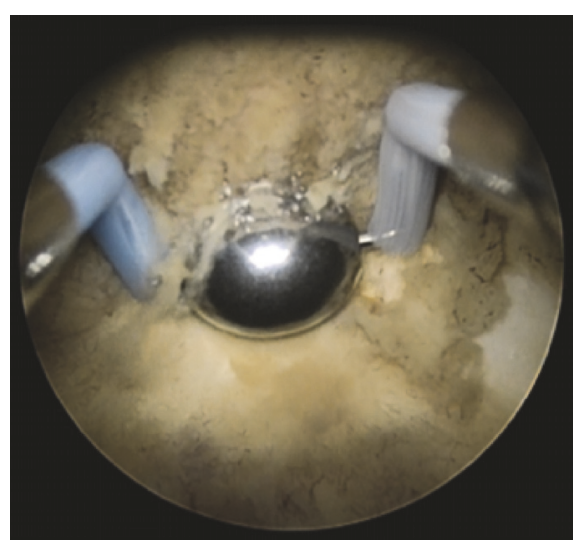

(d)

Figure 2: (a) Hysteroscopic view at high perfusion pressure. (b) Hysteroscopic view at low perfusion pressure with bulging of cystic adenoma seen. (c) Hysteroscopic dissection of cystic adenomyoma wall away from endometrium. (d) Roller ball ablation of adenomyotic deposits.

to be abandoned because the lesion was too firmly adherent to the myometrium, without a well-defined cleavage plane, in contrast to the situation of a myoma. Consequently, the cyst wall, including the yellow-brown deposits representing the ectopic endometriotic deposits (Figure 2(d)), was ablated under ultrasound guidance with the use of a roller ball diathermy.

\section{Conclusion}

Many treatment modalities are now available for the treatment of adenomyosis. The management plan ought to be individualized, depending on the presenting symptom and the desire to achieve a successful pregnancy. Recent development in various nonsurgical and surgical options has significantly improved the prospect of a successful treatment in women wishing to conceive again.

\section{Conflicts of Interest}

The authors declare that there are no conflicts of interest regarding the publication of this paper.

\section{Authors' Contributions}

Jin-Jiao Li and Jacqueline P. W. Chung contributed equally to the manuscript.

\section{Acknowledgments}

This work was supported by grants from the National Natural Science Foundation of China (no. 81270680, no. 81571412) and the Beijing Municipal Administration of Hospital Clinical Medicine, Development of Special Funding Support (ZYLX201406).

\section{References}

[1] P. Vercellini, F. Parazzini, S. Oldani, S. Panazza, T. Bramante, and P. G. Crosignani, "Surgery: Adenomyosis at hysterectomy: a study on frequency distribution and patient characteristics," Human Reproduction, vol. 10, no. 5, pp. 1160-1162, 1995.

[2] D. Vavilis, T. Agorastos, J. Tzafetas et al., "Adenomyosis at hysterectomy: prevalence and relationship to operative findings and reproductive and menstrual factors," Clinical and Experimental Obstetrics \& Gynecology, vol. 24, no. 1, pp. 36-38, 1997. 
[3] J. D. Seidman and K. H. Kjerulff, "Pathologic findings from the Maryland Women's Health Study: Practice patterns in the diagnosis of adenomyosis," International Journal of Gynecological Pathology, vol. 15, no. 3, pp. 217-221, 1996.

[4] F. Parazzini, P. Vercellini, S. Panazza, L. Chatenoud, S. Oldani, and P. G. Crosignani, "Risk factors for adenomyosis," Human Reproduction, vol. 12, no. 6, pp. 1275-1279, 1997.

[5] T. Bergholt, L. Eriksen, N. Berendt, M. Jacobsen, and J. B. Hertz, "Prevalence and risk factors of adenomyosis at hysterectomy," Human Reproduction, vol. 16, no. 11, pp. 2418-2421, 2001.

[6] N. M. de Souza, J. J. Brosens, J. E. Schwieso, T. Paraschos, and R. M. L. Winston, "The potential value of magnetic resonance imaging in infertility," Clinical Radiology, vol. 50, no. 2, pp. 7579, 1995.

[7] G. Kunz, D. Beil, P. Huppert, M. Noe, S. Kissler, and G. Leyendecker, "Adenomyosis in endometriosis-prevalence and impact on fertility: evidence from magnetic resonance imaging," Human Reproduction, vol. 20, no. 8, pp. 2309-2316, 2005.

[8] S. Kissler, S. Zangos, J. Kohl et al., "Duration of dysmenorrhoea and extent of adenomyosis visualised by magnetic resonance imaging," European Journal of Obstetrics \& Gynecology and Reproductive Biology, vol. 137, no. 2, pp. 204-209, 2008.

[9] J. Naftalin, W. Hoo, K. Pateman, D. Mavrelos, T. Holland, and D. Jurkovic, "How common is adenomyosis? A prospective study of prevalence using transvaginal ultrasound in a gynaecology clinic," Human Reproduction, vol. 27, no. 12, pp. 3432-3439, 2012.

[10] N. Di Donato, G. Montanari, A. Benfenati et al., "Prevalence of adenomyosis in women undergoing surgery for endometriosis," European Journal of Obstetrics \& Gynecology and Reproductive Biology, vol. 181, pp. 289-293, 2014.

[11] C. C. Bird, T. W. McElin, and P. Manalo-Estrella, "The elusive adenomyosis of the uterus-revisited," American Journal of Obstetrics \& Gynecology, vol. 112, no. 5, pp. 583-593, 1972.

[12] G. Alabiso, L. Alio, S. Arena et al., "Adenomyosis: What the Patient Needs," Journal of Minimally Invasive Gynecology, vol. 23, no. 4, pp. 476-488, 2016.

[13] G. Leyendecker, A. Bilgicyildirim, M. Inacker et al., "Adenomyosis and endometriosis. Re-visiting their association and further insights into the mechanisms of auto-traumatisation. An MRI study," Archives of Gynecology and Obstetrics, vol. 291, no. 4, pp. 917-932, 2015.

[14] J. Struble, S. Reid, and M. A. Bedaiwy, "Adenomyosis: a clinical review of a challenging gynecologic condition," Journal of Minimally Invasive Gynecology, vol. 23, no. 2, pp. 164-185, 2016.

[15] G. Benagiano, I. Brosens, and M. Habiba, "Structural and molecular features of the endomyometrium in endometriosis and adenomyosis," Human Reproduction Update, vol. 20, no. 3, Article ID dmt052, pp. 386-402, 2014.

[16] Y. Zhang, L. Zhou, T. C. Li, H. Duan, P. Yu, and H. Y. Wang, "Ultrastructural features of endometrial-myometrial interface and its alteration in adenomyosis," International Journal of Clinical and Experimental Pathology, vol. 7, no. 4, pp. 1469-1477, 2014.

[17] H. Takeuchi, M. Kitade, I. Kikuchi, J. Kumakiri, K. Kuroda, and M. Jinushi, "Diagnosis, laparoscopic management, and histopathologic findings of juvenile cystic adenomyoma: A review of nine cases," Fertility and Sterility, vol. 94, no. 3, pp. 862-868, 2010.

[18] M. Levgur, M. A. Abadi, and A. Tucker, "Adenomyosis: symptoms, histology, and pregnancy terminations," Obstetrics \& Gynecology, vol. 95, no. 5, pp. 688-691, 2000.
[19] O. Ozdegirmenci, F. Kayikcioglu, M. A. Akgul et al., "Comparison of levonorgestrel intrauterine system versus hysterectomy on efficacy and quality of life in patients with adenomyosis," Fertility and Sterility, vol. 95, no. 2, pp. 497-502, 2011.

[20] R. C. Benson and V. D. Sneeden, "Adenomyosis: A reappraisal of symptomatology," American Journal of Obstetrics \& Gynecology, vol. 76, no. 5, pp. 1044-1061, 1958.

[21] S. Campo, V. Campo, and G. Benagiano, "Adenomyosis and infertility," Reproductive BioMedicine Online, vol. 24, no. 1, pp. 35-46, 2012.

[22] G. Kunz and G. Leyendecker, "Uterine peristaltic activity during the menstrual cycle: characterization, regulation, function and dysfunction.", Reproductive BioMedicine Online, vol. 4, pp. 5-9, 2002.

[23] G. Benagiano, M. Habiba, and I. Brosens, "The pathophysiology of uterine adenomyosis: An update," Fertility and Sterility, vol. 98, no. 3, pp. 572-579, 2012.

[24] K. Tremellen and P. Russell, "Adenomyosis is a potential cause of recurrent implantation failure during IVF treatment," Australian and New Zealand Journal of Obstetrics and Gynaecology, vol. 51, no. 3, pp. 280-283, 2011.

[25] C. Reinhold, M. Atri, A. Mehio, R. Zakarian, A. E. Aldis, and P. M. Bret, "Diffuse uterine adenomyosis: morphologic criteria and diagnostic accuracy of endovaginal sonography," Radiology, vol. 197, no. 3, pp. 609-614, 1995.

[26] M. Dueholm and E. Lundorf, "Transvaginal ultrasound or MRI for diagnosis of adenomyosis," Current Opinion in Obstetrics and Gynecology, vol. 19, no. 6, pp. 505-512, 2007.

[27] C.-H. Chiang, M.-Y. Chang, J.-J. Hsu et al., "Tumor vascular pattern and blood flow impedance in the differential diagnosis of leiomyoma and adenomyosis by color Doppler sonography," Journal of Assisted Reproduction and Genetics, vol. 16, no. 5, pp. 268-275, 1999.

[28] T. Van den Bosch, M. Dueholm, F. P. Leone et al., "Terms, definitions and measurements to describe sonographic features of myometrium and uterine masses: a consensus opinion from the Morphological Uterus Sonographic Assessment (MUSA) group," Ultrasound in Obstetetrics Gynecology, vol. 46, no. 3, pp. 284-298, 2015.

[29] S. H. Saravelos, K. Jayaprakasan, K. Ojha, and T.-C. Li, "Assessment of the uterus with three-dimensional ultrasound in women undergoing ART," Human Reproduction Update, vol. 23, no. 2, pp. 188-210, 2017.

[30] C. Exacoustos, L. Brienza, and A. Di Giovanni, "Adenomyosis: three-dimensional sonographic findings of the JZ and correlation with histology," Ultrasound in Obstetetrics \& Gynecology, vol. 37, no. 4, pp. 471-479, 2011.

[31] F. Ahmadi and H. Haghighi, "Three-dimensional ultrasound manifestations of adenomyosis," Iranian Journal of Reprodive Medicine, vol. 11, no. 10, pp. 847-848, 2013.

[32] C. Reinhold, F. Tafazoli, A. Mehio et al., "Uterine adenomyosis: Endovaginal US and MR imaging features with histopathologic correlation," RadioGraphics, vol. 19, pp. S147-S160, 1999.

[33] C. Reinhold, S. McCarthy, P. M. Bret et al., "Diffuse adenomyosis: comparison of endovaginal US and MR imaging with histopathologic correlation," Radiology, vol. 199, no. 1, pp. 151158, 1996.

[34] R. Champaneria, P. Abedin, J. Daniels, M. Balogun, and K. S. Khan, "Ultrasound scan and magnetic resonance imaging for the diagnosis of adenomyosis: Systematic review comparing test accuracy," Acta Obstetricia et Gynecologica Scandinavica, vol. 89, no. 11, pp. 1374-1384, 2010. 
[35] S. Acar, E. Millar, M. Mitkova, and V. Mitkov, "Value of ultrasound shear wave elastography in the diagnosis of adenomyosis," Ultrasound, vol. 24, no. 4, pp. 205-213, 2016.

[36] S. R. Soares, M. M. B. B. Dos Reis, and A. F. Camargos, "Diagnostic accuracy of sonohysterography, transvaginal sonography, and hysterosalpingography in patients with uterine cavity diseases," Fertility and Sterility, vol. 73, no. 2, pp. 406-411, 2000.

[37] C. R. Molinas and R. Campo, "Office hysteroscopy and adenomyosis," Best Practice \& Research Clinical Obstetrics \& Gynaecology, vol. 20, no. 4, pp. 557-567, 2006.

[38] A. M. McCausland, "Hysteroscopic myometrial biopsy: Its use in diagnosing adenomyosis and its clinical application," American Journal of Obstetrics \& Gynecology, vol. 166, no. 6 I, pp. 1619-1628, 1992.

[39] A. M. Darwish, A. M. Makhlouf, A. A. Youssof, and H. A. Gadalla, "Hysteroscopic myometrial biopsy in unexplained abnormal uterine bleeding," European Journal of Obstetrics \& Gynecology and Reproductive Biology, vol. 86, no. 2, pp. 139-143, 1999.

[40] L. W. Popp, J. P. Schwiedessen, and R. Gaetje, "Myometrial biopsy in the diagnosis of adenomyosis uteri," American Journal of Obstetrics Gynecology, vol. 169, p. 546, 1993.

[41] S. Gordts, R. Campo, and I. Brosens, "Hysteroscopic diagnosis and excision of myometrial cystic adenomyosis," Journal of Gynecologic Surgery, vol. 11, no. 4, pp. 273-278, 2014.

[42] C.-J. Jeng, S.-H. Huang, J. Shen, C.-S. Chou, and C.-R. Tzeng, "Laparoscopy-guided myometrial biopsy in the definite diagnosis of diffuse adenomyosis," Human Reproduction, vol. 22, no. 7, pp. 2016-2019, 2007.

[43] J. C. Gambone, B. S. Mittman, M. G. Munro, A. R. Scialli, and C. A. Winkel, "Consensus statement for the management of chronic pelvic pain and endometriosis: proceedings of an expert-panel consensus process," Fertility and Sterility, vol. 78, no. 5, pp. 961-972, 2002.

[44] P. Vercellini, G. Frontino, O. De Giorgi, G. Pietropaolo, R. Pasin, and P. G. Crosignani, "Continuous use of an oral contraceptive for endometriosis-associated recurrent dysmenorrhea that does not respond to a cyclic pill regimen," Fertility and Sterility, vol. 80, no. 3, pp. 560-563, 2003.

[45] R. Mansouri, X. M. Santos, J. L. Bercaw-Pratt, and J. E. Dietrich, "Regression of Adenomyosis on Magnetic Resonance Imaging after a Course of Hormonal Suppression in Adolescents: A Case Series," Journal of Pediatric \& Adolescent Gynecology, vol. 28, no. 6, pp. 437-440, 2015.

[46] M. Igarashi, M. Fukuda, A. Ando et al., "Local administration of danazol on pelvic endometriosis and uterine adenomyosis, Nihon Rinsho," Japanese Journal of Clinical Medicine, vol. 59, supplement 1, pp. 153-156, 2001.

[47] M. Igarashi, Y. Abe, M. Fukuda et al., "Erratum: Novel conservative medical therapy for uterine adenomyosis with a danazolloaded intrauterine device (Fertility and Sterility (2000) 74 (412413))," Fertility and Sterility, vol. 74, no. 4, p. 851, 2000.

[48] M. Fawzy and Y. Mesbah, "Comparison of dienogest versus triptorelin acetate in premenopausal women with adenomyosis: a prospective clinical trial," Archives of Gynecology and Obstetrics, vol. 292, no. 6, pp. 1267-1271, 2015.

[49] F. Ji, X. H. Yang, A. L. Ai Xing, T. X. Zi, Y. He, and Y. Ding, "Role of levonorgestrel-releasing intrauterine system in dysmenorrhea due to adenomyosis and the influence on ovarian function," Clinical and Experimental Obstetrics \& Gynecology, vol. 41, no. 6, pp. 677-680, 2014.
[50] O. M. Shaaban, M. K. Ali, A. M. A. Sabra, and D. E. M. Abd El Aal, "Levonorgestrel-releasing intrauterine system versus a lowdose combined oral contraceptive for treatment of adenomyotic uteri: A randomized clinical trial," Contraception, vol. 92, no. 4, pp. 301-307, 2015.

[51] P. Zhang, K. Song, L. Li, K. Yukuwa, and B. Kong, "Efficacy of combined levonorgestrel-releasing intrauterine system with gonadotropin-releasing hormone analog for the treatment of adenomyosis," Medical Principles and Practice, vol. 22, no. 5, pp. 480-483, 2013.

[52] J. Zheng, E. Xia, T. C. Li, and X. Sun, "Comparison of combined transcervical resection of the endometrium and levonorgestrelcontaining intrauterine system treatment versus levonorgestrelcontaining intrauterine system treatment alone in women with adenomyosis: A prospective clinical trial," The Journal of Reproductive Medicine, vol. 58, no. 7-8, pp. 285-290, 2013.

[53] F.-J. Huang, F.-T. Kung, S.-Y. Chang, and T.-Y. Hsu, "Effects of short-course buserelin therapy on adenomyosis: A report of two cases," Obstetrics, Gynaecology and Reproductive Medicine, vol. 44, no. 8, pp. 741-744, 1999.

[54] K. H. Tsui, W. L. Lee, C. Y. Chen et al., "Medical treatment for adenomyosis and/or adenomyoma," Taiwanese Journal of Obestetrics \& Gynecology, vol. 53, no. 4, pp. 459-465, 2014.

[55] J. Kulak Jr., C. Fischer, B. Komm, and H. S. Taylor, "Treatment with bazedoxifene, a selective estrogen receptor modulator, causes regression of endometriosis in a mouse model," Endocrinology, vol. 152, no. 8, pp. 3226-3232, 2011.

[56] A. M. Badawy, A. M. Elnashar, and A. A. Mosbah, "Aromatase inhibitors or gonadotropin-releasing hormone agonists for the management of uterine adenomyosis: A randomized controlled trial," Acta Obstetricia et Gynecologica Scandinavica, vol. 91, no. 4, pp. 489-495, 2012.

[57] F. Kimura, K. Takahashi, K. Takebayashi et al., "Concomitant treatment of severe uterine adenomyosis in a premenopausal woman with an aromatase inhibitor and a gonadotropinreleasing hormone agonist," Fertility and Sterility, vol. 87, no. 6, pp. 1468-e9, 2007.

[58] T. Kalampokas, M. Kamath, I. Boutas, and E. Kalampokas, "Ulipristal acetate for uterine fibroids: A systematic review and meta-analysis," Gynecological Endocrinology, vol. 32, no. 2, pp. 91-96, 2016.

[59] J. Donnez and M.-M. Dolmans, "Uterine fibroid management: From the present to the future," Human Reproduction Update, vol. 22, no. 6, pp. 665-686, 2016.

[60] B. Zhu, Y. Chen, X. Shen, X. Liu, and S.-W. Guo, "Anti-platelet therapy holds promises in treating adenomyosis: Experimental evidence," Reproductive Biology and Endocrinology, vol. 14, no. 1, article no. 66, 2016.

[61] M. Popovic, S. Puchner, D. Berzaczy, J. Lammer, and R. A. Bucek, "Uterine artery embolization for the treatment of adenomyosis: A review," Journal of Vascular and Interventional Radiology, vol. 22, no. 7, pp. 901-909, 2011.

[62] J. Zhou, L. He, P. Liu et al., "Outcomes in adenomyosis treated with uterine artery embolization are associated with lesion vascularity: A long-term follow-up study of 252 cases," PLoS ONE, vol. 11, no. 11, Article ID e0165610, 2016.

[63] X. Dong and Z. Yang, "High-intensity focused ultrasound ablation of uterine localized adenomyosis," Current Opinion in Obstetrics and Gynecology, vol. 22, no. 4, pp. 326-330, 2010.

[64] M. Zhou, J.-Y. Chen, L.-D. Tang, W.-Z. Chen, and Z.-B. Wang, "Ultrasound-guided high-intensity focused ultrasound ablation 
for adenomyosis: The clinical experience of a single center," Fertility and Sterility, vol. 95, no. 3, pp. 900-905, 2011.

[65] L. Zhang, W. Zhang, F. Orsi, W. Chen, and Z. Wang, "Ultrasound-guided high intensity focused ultrasound for the treatment of gynaecological diseases: A review of safety and efficacy," International Journal of Hyperthermia, vol. 31, no. 3, pp. 280284, 2015.

[66] V. McCausland and A. McCausland, "The response of adenomyosis to endometrial ablation/resection," Human Reproduction Update, vol. 4, no. 4, pp. 350-359, 1998.

[67] C. Wood, "Surgical and medical treatment of adenomyosis," Human Reproduction Update, vol. 4, no. 4, pp. 323-336, 1998.

[68] D. R. Phillips, H. G. Nathanson, S. J. Milim, and J. S. Haselkorn, "Laparoscopic bipolar coagulation for the conservative treatment of adenomyomata," The Journal of Minimally Invasive Gynecology, vol. 4, no. 1, pp. 19-24, 1996.

[69] M. Furuhashi, Y. Miyabe, Y. Katsumata, H. Oda, and N. Imai, "Comparison of complications of vaginal hysterectomy in patients with leiomyomas and in patients with adenomyosis," Archives of Gynecology and Obstetrics, vol. 262, no. 1-2, pp. 6973, 1998.

[70] T. G. Stovall, F. W. Ling, and D. A. Crawford, "Hysterectomy for chronic pelvic pain of presumed uterine etiology," Obstetrics \& Gynecology, vol. 75, no. 4, pp. 676-679, 1990.

[71] L. J. Orozco, M. Tristan, M. M. T. Vreugdenhil, and A. Salazar, "Hysterectomy versus hysterectomy plus oophorectomy for premenopausal women," Cochrane Database of Systematic Reviews, vol. 7, p. CD005638, 2014.

[72] E. C. Evans, K. A. Matteson, F. J. Orejuela et al., "Salpingooophorectomy at the Time of Benign Hysterectomy: A Systematic Review," Obstetrics \& Gynecology, vol. 128, no. 3, pp. 476485, 2016.

[73] V. C. Kok, H.-J. Tsai, C.-F. Su, and C.-K. Lee, “The risks for ovarian, endometrial, breast, colorectal, and other cancers in women with newly diagnosed endometriosis or adenomyosis: A population-based study," International Journal of Gynecological Cancer, vol. 25, no. 6, pp. 968-976, 2015.

[74] P. Vercellini, D. Consonni, D. Dridi, B. Bracco, M. P. Frattaruolo, and E. Somigliana, "Uterine adenomyosis and in vitro fertilization outcome: A systematic review and meta-analysis," Human Reproduction, vol. 29, no. 5, pp. 964-977, 2014.

[75] V. Thalluri and K. P. Tremellen, "Ultrasound diagnosed adenomyosis has a negative impact on successful implantation following GnRH antagonist IVF treatment," Human Reproduction, vol. 27, no. 12, pp. 3487-3492, 2012.

[76] J. M. Puente, A. Fabris, J. Patel et al., "Adenomyosis in infertile women: Prevalence and the role of 3D ultrasound as a marker of severity of the disease," Reproductive Biology and Endocrinology, vol. 14, no. 1, article no. 60, 2016.

[77] J. R. Nelson and S. L. Corson, "Long-term management of adenomyosis with a gonadotropin-releasing hormone agonist: A case report," Fertility and Sterility, vol. 59, no. 2, pp. 441-443, 1993.

[78] P. D. Silva, H. E. Perkins, and C. W. Schauberger, "Live birth after treatment of severe adenomyosis with a gonadotropinreleasing hormone agonist," Fertility and Sterility, vol. 61, no. 1, pp. 171-172, 1994.

[79] L.-M. Zhou, J. Zheng, Y.-T. Sun, Y.-Y. Zhao, and A.-L. Xia, "Study on leuprorelin acetate in treatment of uterine adenomyosis with infertility," Zhonghua Fu Chan Ke Za Zhi, vol. 48, no. 5, pp. 334-337, 2013.
[80] T. Tao, S. Chen, X. Chen et al., "Effects of uterine adenomyosis on clinical outcomes of infertility patients treated with in vitro fertilization/intracytoplasmic sperm injection-embryo transfer (IVF/ICSI-ET)," Nan fang yi ke da xue xue bao = Journal of Southern Medical University, vol. 35, no. 2, pp. 248-251, 2015.

[81] A. Hashimoto, T. Iriyama, and S. Sayama, "Adenomyosis and adverse perinatal outcomes: increased risk of second trimester miscarriage, preeclampsia, and placental malposition," The Journal of Maternal-fetal Neonatal Medicine, vol. 23, no. 1-6, p. 18, 2017.

[82] Z. Niu, Q. Chen, Y. Sun, and Y. Feng, "Long-term pituitary downregulation before frozen embryo transfer could improve pregnancy outcomes in women with adenomyosis," Gynecological Endocrinology, vol. 29, no. 12, pp. 1026-1030, 2013.

[83] O. Moraloglu, E. Tonguc, T. Var, T. Zeyrek, and S. Batioglu, "Treatment with oxytocin antagonists before embryo transfer may increase implantation rates after IVF," Reproductive BioMedicine Online, vol. 21, no. 3, pp. 338-343, 2010.

[84] S.-W. Guo, X. Mao, Q. Ma, and X. Liu, "Dysmenorrhea and its severity are associated with increased uterine contractility and overexpression of oxytocin receptor (OTR) in women with symptomatic adenomyosis," Fertility and Sterility, vol. 99, no. 1, pp. 231-240, 2013.

[85] E. H. Y. Ng, R. H. W. Li, L. Chen, V. T. N. Lan, H. M. Tuong, and S. Quan, "A randomized double blind comparison of atosiban in patients undergoing IVF treatment," Human Reproduction, vol. 29, no. 12, pp. 2687-2694, 2014.

[86] C. Coughlan, W. Ledger, Q. Wang et al., "Recurrent implantation failure: Definition and management," Reproductive BioMedicine Online, vol. 28, no. 1, pp. 14-38, 2014.

[87] Y. Kishi, M. Yabuta, and F. Taniguchi, "Who will benefit from uterus-sparing surgery in adenomyosis-associated subfertility?" Fertility and Sterility, vol. 102, no. 3, pp. 802-e1, 2014.

[88] G. F. Grimbizis, T. Mikos, and B. Tarlatzis, "Uterus-sparing operative treatment for adenomyosis," Fertility and Sterility, vol. 101, no. 2, pp. 472-e8, 2014.

[89] A. S. W. Wong, E. C. W. Cheung, K.-T. Leung, S.-W. Yeung, T.-Y. Leung, and T.-Y. Fung, "Transcervical intralesional vasopressin injection in hysteroscopic myomectomy-description of a new technique," Journal of Laparoendoscopic \& Advanced Surgical Techniques, vol. 23, no. 3, pp. 258-262, 2013.

[90] D. Xu, G. Jamail, M. Xue, X. Guan, and L. Wang, "Removal of Retained Adherent Placental Remnants Using Hysteroscopy Endo-Operative System (HEOS)," Journal of Minimally Invasive Gynecology, vol. 22, no. 6, p. S137, 2015. 


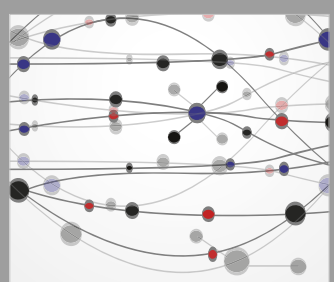

The Scientific World Journal
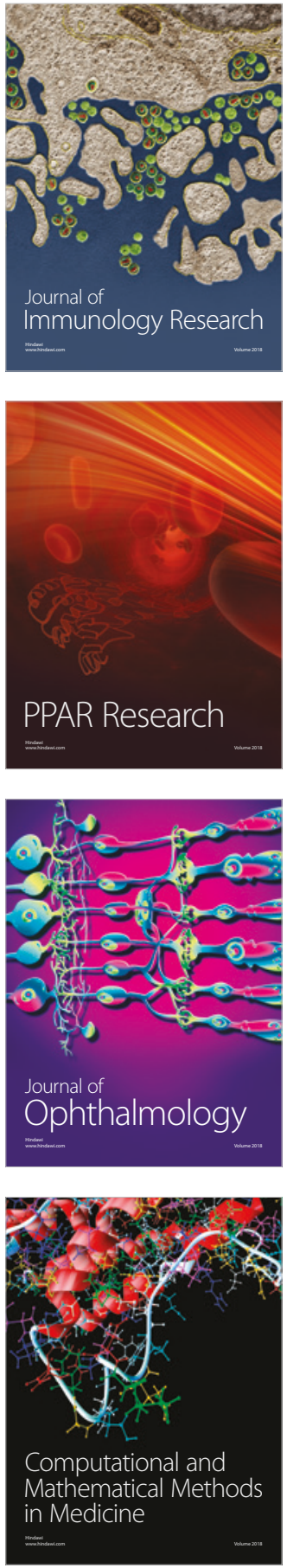

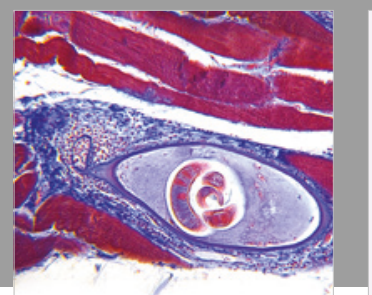

Gastroenterology Research and Practice

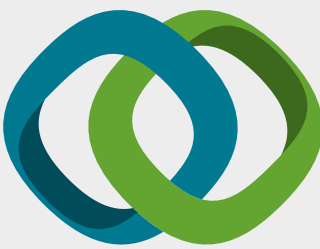

\section{Hindawi}

Submit your manuscripts at

www.hindawi.com
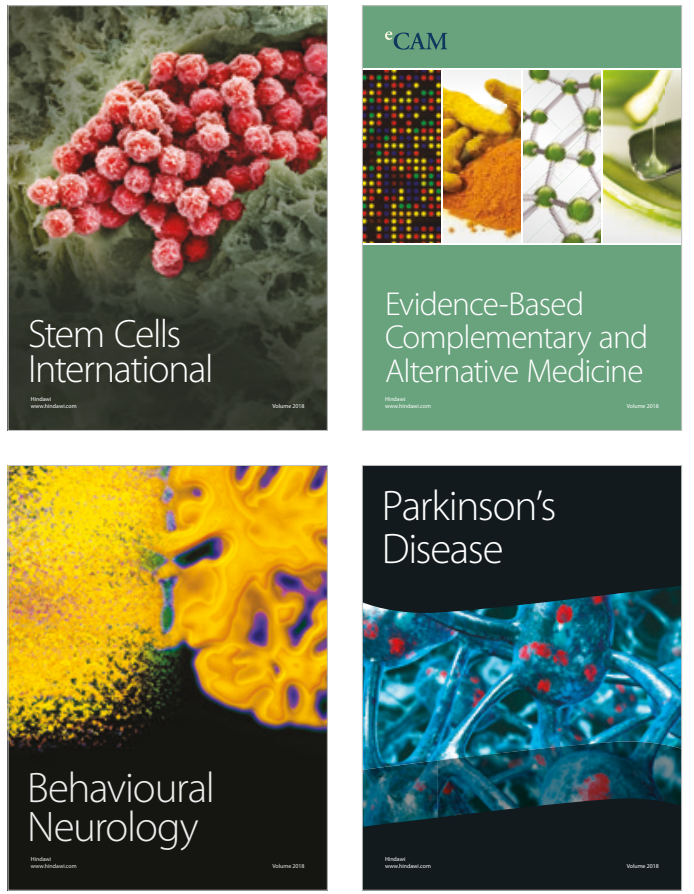

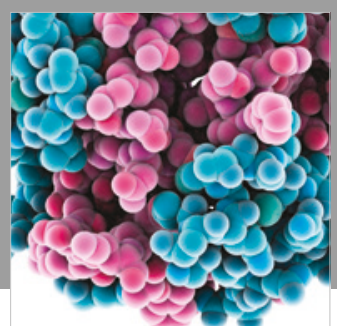

ournal of

Diabetes Research

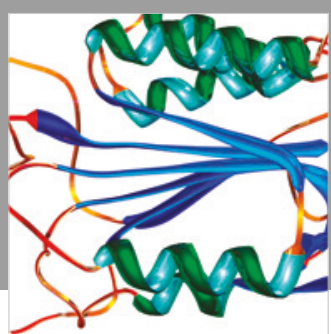

Disease Markers
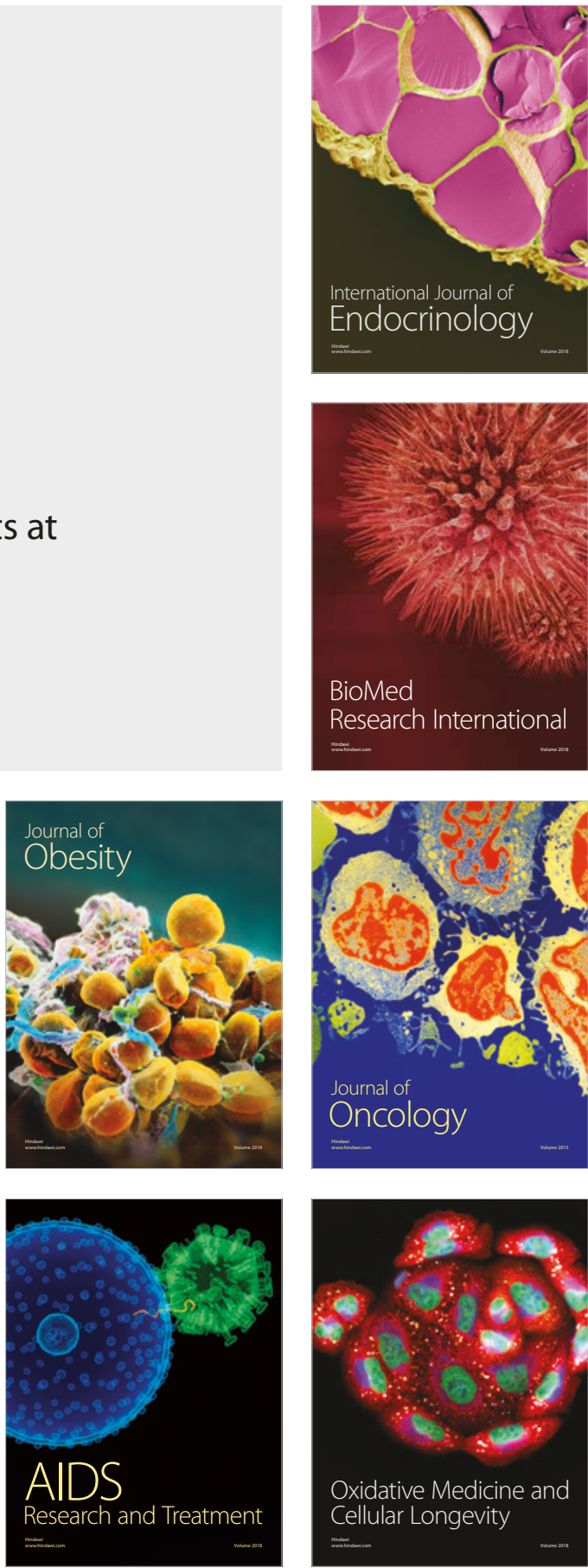